\title{
Spectral investigations of some piperidin-4-one molecular addition compounds
}

\author{
Jayaraman CHAKKARAVARTHY, ${ }^{1}$ Inbasekaran MUTHUVEL,${ }^{2,3}$ and \\ Ganesamoorthy THIRUNARAYANAN ${ }^{* 2}$
}

\author{
${ }^{1}$ Department of Chemistry, Periyar Government Arts College, Cuddalore-607 001, Tamilnadu, India \\ ${ }^{2}$ Department of Chemistry, Annamalai University, Annamalainagar-608 002, Tamilnadu, India \\ ${ }^{3}$ Department of Chemistry, MR Government Arts College, Mannargudi-614 001, Tamilnadu, India
}

\begin{abstract}
In the present study, some 2- and 3-substituted piperidin-4-ones ( $\left.\mathbf{A}_{1}-\mathbf{A}_{3}\right)$ were chosen as proton acceptor and maleic anhydride was chosen as proton donor. Piperidin-4-ones $\left(\mathbf{A}_{\mathbf{1}}, \mathbf{A}_{\mathbf{2}} \boldsymbol{\&} \mathbf{A}_{\mathbf{3}}\right)$ were mixed with maleic anhydride in ether medium and the corresponding molecular adduct products $\left(\mathbf{B}_{1}, \mathbf{B}_{\mathbf{2}} \boldsymbol{\&} \mathbf{B}_{3}\right)$ thus obtained were collected and purified. The ${ }^{1} \mathrm{H}$ and ${ }^{13} \mathrm{C}$ NMR spectra were recorded for piperidine-4-one and their addition compounds. The ${ }^{1} \mathrm{H}$ and ${ }^{13} \mathrm{C}$ NMR chemical shifts of products $\left(\mathbf{B}_{1}, \mathbf{B}_{\mathbf{2}} \boldsymbol{\&} \mathbf{B}_{3}\right)$ are analyzed and compared with those of the corresponding piperidine-4-ones $\left(\mathbf{A}_{1}, \mathbf{A}_{\mathbf{2}} \&\right.$ A3). In order to confirm the formation of molecular addition compounds the GC-Mass spectrum was recorded for all adducts $\mathbf{B}_{1}-\mathbf{B}_{3}$ and the fragmentations patterns were analyzed.
\end{abstract}

Keywords: piperidine-4-ones; molecular addition; steric effect; polar effect; NMR spectra.

\section{Introduction}

Organic chemists and scientists employed NMR spectroscopic data for investigating the structure of organic molecules including conformers, isomers and diastereoisomers. The purpose in which chemists use NMR technique is for investigating the structural diagnosis of organic molecules, making stereo chemical dependent phenomena, such as hydrogen exchange, conformational changes and internal rotation. Stereochemical problems are increasingly being investigated by ${ }^{13} \mathrm{C}$ NMR spectroscopy. The conformational and charge distribution on a molecule was studied by the observed chemical shifts (ppm). Considerable attention has also been focused on the study of substituent effect on ${ }^{13} \mathrm{C}$ chemical shifts.

Lambert et al. [1] investigated the effect of electronegativity of heteroatom in cyclohexanes by chemical shifts (ppm). About 50 ppm of downfield chemical shifts produced by one unit of electronegativity and it causes very small changes alpha and beta carbons in piperidones. The influence of electronegativity of heteroatom in 2, 6-diphenyl-1hetera-4-cyclohexanones was studied by Ramalingam et al. [2]. The benzylic carbon appears downfield in 2, 6-diphenyl-1-hetera-4-cyclohexanones than 3,5diphenylcyclohexanone. The deshielding effect on piperidones are in the order of $\mathrm{O}>\mathrm{N}\left(\mathrm{CH}_{3}\right)_{2}>\mathrm{NH}>\mathrm{S}$. The axial group protons possess smaller alpha and beta effects than an equatorial group. Ravindran et al. [3] investigated the ${ }^{13} \mathrm{C}$ NMR spectra of some $\mathrm{N}$ nitroso-2,6-diphenylpiperidin-4-ones and N-nitroso2,6-diphenylpiperidines. The shielding effect of syn carbon was observed as $7.19 \mathrm{ppm}$ related to the parental amine in N-nitroso-2, 6-diphenylpiperidin-4one. The separate ${ }^{13} \mathrm{C}$ NMR chemical shifts (ppm) of 51.9 and $60.4 \mathrm{ppm}$ inferred as syn- $\beta$ and anti- $\beta$ benzylic carbons. The chemical shifts of protons are affected by electronic effects such as resonance, inductive, polar, steric, field, magnetic anisotropic and hyper conjugative effects. The inductive effect of halogen atom plays an important role for determining the chemical shifts of protons in methyl halides [4]. Numerous illustrations were reported for the significant downfield chemical shifts of protons and are close proximity to other groups [5-9]. Diamagnetic anisotropy arising due to the ring current effect operates in aromatic compounds. The $\delta$-value (7.25 ppm) of the protons in benzene itself is an evidence of a considerable deshielding of the protons in the plane of the ring. Protons held above the plane of the aromatic nucleus will be shielded. Aldehydic protons resonate at very low field, since they deshielded in the region of carbonyl carbons. Similarly, in benzaldehyde the ortho protons to the formyl group absorb at a slightly lower field $(\delta 7.72$ ppm) than the other three aromatic protons [4] $(\delta 7.41$ ppm). The equatorial configuration [10] of 3-methyl group in 3-methyl-2,6-diphenylpiperiding-4-one was determined on the basis of the observed benzylic proton chemical shift at C-2 absorbs at a higher field ( $\delta$ $4.1 \mathrm{ppm})$. Whether the methyl group in axial configuration, the proton chemical shift absorbs at C2 should lower field than C-6. Several substituted Nchloro [11], N-methyl [12], N-benzoyl [13], N-

\footnotetext{
* Corresponding author. E-mail addresses: thirunarayanan.g.10313@annamalaiuniversity.ac.in; drgtnarayanan@gmail.com (Ganesamoorthy Thirunarayanan).
} 
propanoyl and N-p-toluene sulphonyl [14], N-acetyl [15] piperidones have been prepared and characterized by their NMR data. A survey of literature reveals that much attention has been paid on the chemistry of ${ }^{13} \mathrm{C}$ and ${ }^{1} \mathrm{H}$ NMR of piperidones and their derivatives. The present study was focused on the NMR analysis of some molecular addition compounds derived from 2,6-diphenylpiperidin-4ones.

\section{Experimental}

\subsection{Materials and methods}

In this investigation, all chemicals were procured from Sigma-Aldrich Chemical Company. The melting point of the piperidones were determined in Guna melting point apparatus and are uncorrected. Infrared spectra of addition compounds were recorded in Shimadzu-1650 FT-IR spectrophotometer $\left(4000-400 \mathrm{~cm}^{-1}\right)$ with $\mathrm{KBr}$ pellets. The NMR spectra of all compounds were recorded on Bruker NMR spectrometer operating at 200 $\mathrm{MHz}$ for ${ }^{1} \mathrm{H}$ and $50.3 \mathrm{MHz}$ for ${ }^{13} \mathrm{C}$ spectra, dissolving 50 $\mathrm{mg}$ of the sample in $0.5 \mathrm{ml}$ of $\mathrm{d}_{6}$ DMSO solvent using TMS as internal standard. Mass spectral fragments of synthesized piperidones were collected from JEOL JMS600H Mass Spectrometer. The elemental analysis of the unknown compounds was performed in Thermo Finnigan elemental analyzer.

\subsection{Typical method for synthesis R(2), C(6)- diphenylpiperidn-4-ones \\ The piperidin-4-ones such as 3-methyl-2,6- diphenylpiperidin-4-one (A1), 3-ethyl-2,6- diphenylpiperidin-4-one (A2) and 2,6-} diphenylpiperidin-4-one (A3) were synthesized and their purities were analyzed using the procedure reported by Noller et al. [16] and Lakshmanan et al. [17]. A solution of ammonium acetate $(0.1 \mathrm{~mol})$ in a mixture of ethanol $(10 \mathrm{~mL})$ and glacial acetic acid $(5 \mathrm{~mL})$ was mixed with the appropriate ketone $(0.1 \mathrm{~mol})$ and benzaldehyde $(0.2$ mol). The mixture was heated until a purple color was developed. After cooling, the mixture was kept aside for one day. The lower layer was treated with ether $(50 \mathrm{~mL})$ and concentrated $\mathrm{HCl}(10 \mathrm{~mL})$. Piperidone hydrochloride was precipitated with ammonia and acetone. The free base was liberated by adding water (15 $\mathrm{mL}$ ), and the obtained piperidones were recrystallized in distilled ethanol.

\subsection{Typical procedure for the preparation of molecular addition compounds}

The molecular addition compounds were prepared by a literature method [18]. $1 \mathrm{~g}$ of various piperidone in anhydrous ether $(5 \mathrm{~mL})$ at room temperature was added to a solution of maleic anhydride $(0.01 \mathrm{~mL})$ in anhydrous ether $(10 \mathrm{~mL})$ with gentle shaking (Scheme $1)$. There is an immediate exothermic reaction and the compound is separated out from the solution in excellent yield and washed the product with ether. The molecular addition compounds of piperidones $\mathbf{A}_{1}, \mathbf{A}_{\mathbf{2}}$ and $\mathbf{A}_{\mathbf{3}}$ are named as $\mathbf{B}_{\mathbf{1}}, \mathbf{B}_{\mathbf{2}}$ and $\mathbf{B}_{\mathbf{3}}$ respectively.

The analytical, physical, infrared and mass spectral data of these molecular addition compounds are as follows.
Compound $\left(\mathbf{B}_{1}\right), \mathbf{R}=\mathbf{C H}_{3}$ : light yellow solid; $\mathrm{m}$. p. $116-117^{\circ} \mathrm{C}$; IR $\left(4000-400, \mathrm{~cm}^{-1}\right) v=3488,3325,3012$, 2998, 1678, 1643, 1532, 738, 432; Mass (m/z): 381[ $\left.\mathrm{M}^{+}\right]$, $363\left[\mathrm{M}-\mathrm{H}_{2} \mathrm{O}\right]^{+}, 335,292,265,195,187,132,104,91$, 78, 51. M.F. $\mathrm{C}_{22} \mathrm{H}_{23} \mathrm{NO}_{5}$; M.W. 381; Anal. (\%) - found: $69.30 \mathrm{C}, 6.01 \mathrm{H}, 3.60 \mathrm{~N}$; calculated: $69.28 \mathrm{C}, 6.08 \mathrm{H}$, $3.67 \mathrm{~N}$.

Compound ( $\left.\mathbf{B}_{2}\right), \mathbf{R}=\mathbf{C}_{2} \mathbf{H}_{5}$ : Curd white solid, m. p. $132-133{ }^{\circ} \mathrm{C}$; IR $\left(4000-400, \mathrm{~cm}^{-1}\right) v=3503,3475,3098$,

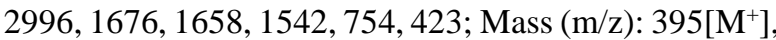
377[M- $\left.\mathrm{M}_{2} \mathrm{O}\right]^{+}, 349,306,279,201,132,104,91,78,51$, 29, 15, 14; M.F. $\mathrm{C}_{23} \mathrm{H}_{25} \mathrm{NO}_{5}$; M.W. 395; Anal. (\%) found: $69.92 \mathrm{C}, 6.30 \mathrm{H}, 3.50 \mathrm{~N}$; calculated: $69.86 \mathrm{C}$, $6.37 \mathrm{H}, 3.54 \mathrm{~N}$.

Compound $\left(\mathbf{B}_{3}\right), \mathbf{R}=\mathbf{H}$ : Pale yellow solid, m. p. 122 $123^{\circ} \mathrm{C}$; IR $\left(4000-400, \mathrm{~cm}^{-1}\right) \mathrm{v}=3516,3445,3018,2990$, 1683, 1643, 1510, 773, 481; Mass (m/z): 367[ $\left.\mathrm{M}^{+}\right]$, $349\left[\mathrm{M}-\mathrm{H}_{2} \mathrm{O}\right]^{+}, 321,278,251,195,173,122,104,91,77$, 51; M.F. $\mathrm{C}_{21} \mathrm{H}_{21} \mathrm{NO}_{5}$; M.W. 367; Anal. (\%) - found: 668.68 C, $5.69 \mathrm{H}, 3.76 \mathrm{~N}$; calculated: $68.65 \mathrm{C}, 5.76 \mathrm{H}$, $3.81 \mathrm{~N}$.

\section{Results and discussion}

A molecular addition compound contains all the masses of the two reactants and it results from simply the addition of two reactants in a particular solvent. Electron donor - acceptor compound is one of the important classes of molecular addition compounds. For example, the picrate addition compounds formed by picric acid with many aromatic hydrocarbons and amines. Olefins behaved as acceptor molecules when they possess electron withdrawing substituents. It has been previously reported [19] that succinic anhydride forms $\mathrm{N}$-phenyl succinimide with aniline. We thought that maleic anhydride also should behave similar to succinic anhydride in forming derivatives of these types. A review of literature reveals that maleic anhydride takes part in a nuclear addition reaction with diene (Diels Alder reaction). This prompted us to study the reactions of maleic anhydride with some piperidin-4-ones. Piperidin-4-ones are expected to behave as electron donors (due to nitrogen has lone pair electrons) and the maleic anhydride is electron acceptor. Therefore, one can expect molecular addition compounds should result from mixing of maleic anhydride with piperidin-4-ones. The other possibility is that the maleic anhydride may add to $\mathrm{NH}$ bond and results $\mathrm{N}-3$-carboxypropenoyl derivative.

In the present study 3-methyl-2,6-diphenylpiperidin4-one (A1), 3-ethy1-2,6-diphenylpiperidin-4-one ( $\left.\mathbf{A}_{2}\right)$ and 2,6-diphenylpiperidin-4-one (A) were chosen and the reaction with maleic anhydride was carried out. Piperidin-4-ones were mixed with maleic anhydride in ether medium and the products thus obtained were collected, purified and analyzed in detail. Supposing that the NMR data were not reported in previously published paper [16] we can consider that the compounds are new. Therefore, the assigned NMR chemical shifts (ppm) of these compounds were compared with literature data.

If the product is N-3-carboxypropanoyl derivative, then one can expect two different signals for ethylenic protons at $\mathrm{N}$-substituent in the ${ }^{1} \mathrm{H}$ NMR spectrum. 
However only one signal is obtained for ethylenic protons in ${ }^{1} \mathrm{H}$ NMR spectrum. Moreover, a review of literature reveals that abnormal vicinal coupling constants are observed for both $\mathbf{J}_{\text {trans }}$ and $\mathbf{J}_{\text {cis }}$ in $\mathrm{N}$-acetyl derivatives and these derivatives mainly adopt conformations other than normal chair conformations. However, according to our current investigations, the coupling constant values of $\mathrm{J}_{\text {trans }}$ and $\mathrm{J}_{\text {cis }}$ of $\mathrm{C}(2)-\mathrm{C}(3)$ and $\mathrm{C}(5)-\mathrm{C}(6)$ bonds are agreeable with the expected values for substituents in equatorial orientations with chair conformation. Therefore, the products formed in the present study are different from that of N-3carboxypropenoy1 derivative. Probably the maleic acid forms addition compound (acid - base additive) with piperidin-4-ones as shown in Scheme 1.

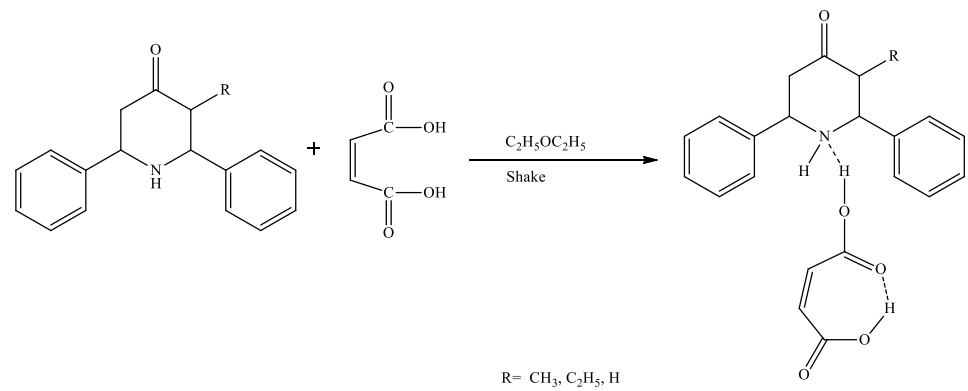

Scheme 1. Preparation of molecular addition compounds from 3-substituted 2,4-diphenylpiperidin-4-ones

The formed compounds may be similar to piperidone hydrochloride. The ${ }^{1} \mathrm{H}$ NMR data revealed considerable deshielding magnitude for all heterocyclic protons and ${ }^{13} \mathrm{C}$ NMR data revealed considerable shielding magnitude for all the compounds. Therefore, it is reasonably assumed that the formed compounds structures are as shown in Figure 1.

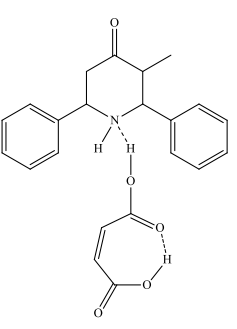

$\mathbf{B}_{1}$

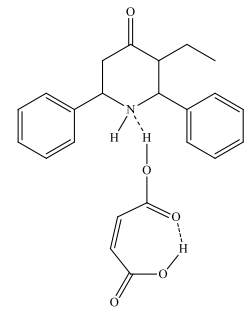

$\mathrm{B}_{2}$

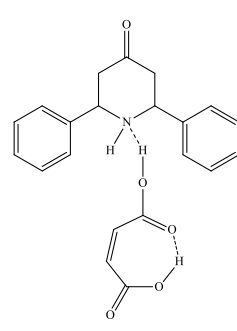

Figure 1. Structure of the molecular addition compounds $\mathbf{B}_{\mathbf{1}}$, $\mathbf{B}_{2}$ and $\mathbf{B}_{3}$

\section{1. ${ }^{1} \mathrm{H}$ NMR spectral analysis}

Recorded ${ }^{1} \mathrm{H}$ NMR spectra of molecular addition compounds $\mathbf{B}_{1}, \mathbf{B}_{\mathbf{2}}$ and $\mathbf{B}_{\mathbf{3}}$ are shown in Figures 2-4.

The observed chemical shifts of molecular addition substrates $\mathbf{B}_{1}, \mathbf{B}_{\mathbf{2}}$ and $\mathbf{B}_{\mathbf{3}}$ are compared with their parent piperidones $\mathbf{A}_{1}, \mathbf{A}_{2}$ and $\mathbf{A}_{3}$ and such a comparison was tabulated in Table 1.

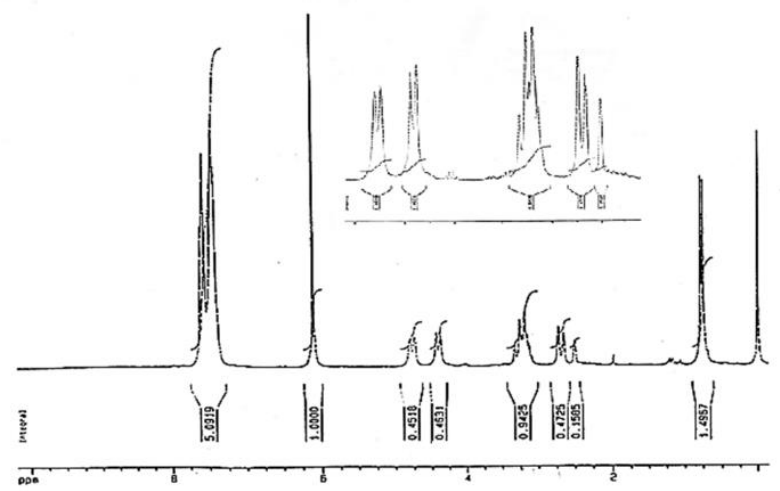

Figure 2. ${ }^{1} \mathrm{H}$ NMR spectrum of molecular addition compound $\mathbf{B}_{1}$

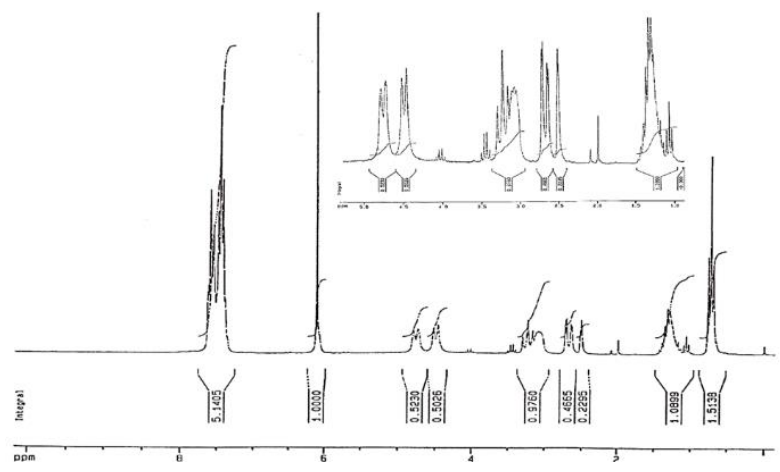

Figure 3. ${ }^{1} \mathrm{H}$ NMR spectrum of molecular addition compound $\mathbf{B}_{2}$

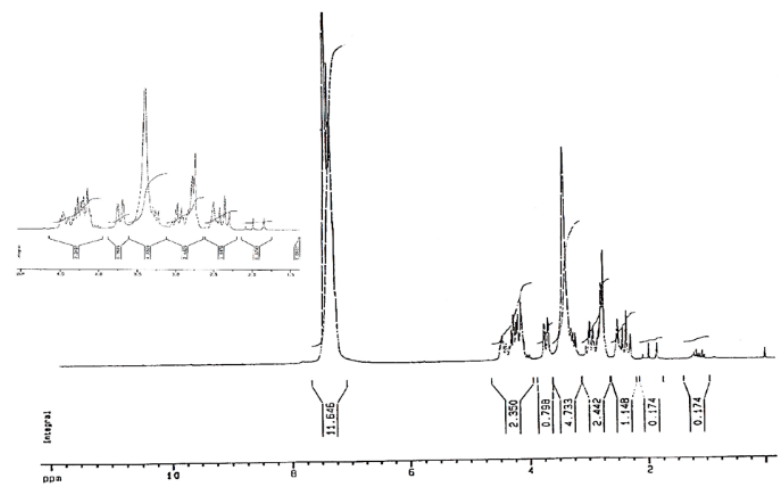

Figure 4. ${ }^{1} \mathrm{H}$ NMR spectrum of molecular addition compound $\mathbf{B}_{3}$

Based on the absorption position, integral and multiplicities of the signals, the chemical shifts were assigned. The aromatic protons absorb around $7 \mathrm{ppm}$. In the ${ }^{1} \mathrm{H}$ NMR spectrum of 3-methy1 compound $\mathbf{B}_{1}$, the observed doublet at $0.77 \mathrm{ppm}$ was assigned for methyl protons at $\mathrm{C}-3$. The coupling constant extracted from the signals is $6.5 \mathrm{~Hz}$. A doublet of doublet obtained at 4.77 and $4.41 \mathrm{ppm}$ along with the coupling constant $11.69 \mathrm{~Hz}$ are assigned for the corresponding benzylic protons at C-6 and C-2 positions. The equatorial configured methylene $\mathrm{C}-5$ proton gave a doublet of doublet at 2.70 ppm with the coupling constants of $13.50 \mathrm{~Hz}$.

The spectrum reveals overlapping of signals for $\mathrm{H}-3$ besides an axial methylene proton of $\mathrm{C}-5$ i.e., $\mathrm{H}_{5}$. From 
the spacing between the lines, it is clear that the outer lines in the downfield region correspond to $\mathrm{H}_{5 \mathrm{a}}$ signal. The high intense signal at $6.11 \mathrm{ppm}$ is due to $\mathrm{CH}$ protons of maleic part of the compound. In a similar manner assignment were made for 3-ethyl compound $\mathbf{B}_{2}$. The spectrum of simple compound $\mathbf{B}_{\mathbf{3}}$ reveals signal gestures for the presence of dual conformers. Due to this the assignment of signal and the calculation of coupling constants are very difficult. The assigned chemical shifts along with coupling constants are tabulated in Table 1.

Assigned proton chemical shifts of 3-ethyl compound $\mathbf{B}_{2}$ are compared with those of 3-ethyl-2, 6diphenylpiperidin-4-one A2. These values are presented in Table 1.

Table 1. The ${ }^{1} \mathrm{H}$ chemical shifts (ppm) of compounds $\mathbf{B}_{\mathbf{1}}-\mathbf{B}_{3}$, and the corresponding parents $\mathbf{A}_{\mathbf{1}}-\mathbf{A}_{\mathbf{3}}$.

\begin{tabular}{|c|c|c|c|c|c|c|c|}
\hline Cpds. & $\mathbf{H}(\mathbf{2})$ & $\mathbf{H}(\mathbf{3})$ & $\mathbf{H}(\mathbf{5 a x})$ & H5(eq) & $\mathbf{H}(6)$ & Alkyl protons & $\begin{array}{l}\text { Other } \\
\text { protons }\end{array}$ \\
\hline $\mathbf{B}_{1}$ & 4.41 & 3.19 & 3.26 & 2.70 & 4.77 & 0.77 & $7.42-7.60$ \\
\hline $\mathbf{B}_{2}$ & 4.49 & $3.10-3.05$ & 3.23 & 2.67 & 4.75 & $\begin{array}{l}0.72\left(\mathrm{CH}_{3}\right) \\
1.42-1.14\left(-\mathrm{CH}_{2}-\right)\end{array}$ & $7.38-7.61$ \\
\hline $\mathbf{A}_{1}$ & 3.63 & - & 2.74 & 2.83 & 4.10 & - & 7.24-7.48 \\
\hline $\mathbf{A}_{2}$ & 3.72 & - & 2.73 & 2.61 & 4.07 & - & - \\
\hline
\end{tabular}

Table 1 reveals that the heterocyclic protons resonate considerably at downfield in the maleic acid product of 3-ethylpiperidone $\mathbf{B}_{2}$. It is also evident that strong interaction exists between the axial lone pair on nitrogen of heterocyclic ring with the proton of maleic acid part in the compound $\mathbf{B}_{2}$.

Piperidone ring protons are deshielded due to the formation of molecular addition compounds, an exception being the equatorial proton of methylene group at $\mathrm{C}-5$ in the 3-methyl addition compound $\mathbf{B}_{1}$. The magnitude of deshielding observed of $\mathrm{H}(2)$ is roughly the same in the 3-methyl addition compound $\mathbf{B}_{\mathbf{1}}(+0.78$ ppm) and 3 ethyl addition compound (+0.77 ppm) $\mathbf{B}_{2}$. The observed deshielding magnitude of $\mathrm{H}(6)$ was slightly lesser than that observed on $\mathrm{H}(2)$.

It is very interesting to note that deshielding magnitude observed on benzylic protons are significantly more than those detected on axial methylene protons of $\mathrm{C}-5(=+0.5 \mathrm{ppm})\left(\mathrm{H}_{5 \mathrm{a}}\right)$. This contrasts with the lesser shielding magnitude observed on benzylic carbons compared to methylene (C-5) and methine carbons (C-3).

In present investigation of the adducts, an axial methylene hydrogen of C-5 and C-3 involves syn 1,3diaxial interaction with the maleic anhydride part of the addition compound. Due to these steric interfaces, an axial protons and carbons of C-3 and C-5 are deshielded. The C-2 and C- 6 hydrogens of benzylic rings were experiencing the steric interaction with the $\mathrm{C}=\mathrm{O}$ of the maleic anhydride of the addition compound. Due to this, the benzylic hydrogens and carbons are shifted to low fields of absorption. In addition, all benzylic hydrogens probably shifted to low field of absorption due to anisotropic effect of carbonyl cluster of the maleic anhydride part of the addition compound. Thus, the benzylic hydrogens are deshielded due to steric interaction with the carbonyl oxygen and due to attractive anisotropy of carbonyl cluster of maleic anhydrides. Both steric and attractive anisotropy are probably responsible and causes of shifting of large magnitude of low field of absorption detected for benzylic protons related to axial methylene protons at C5 .

\section{2. ${ }^{13}$ C NMR spectral analysis}

All ${ }^{13} \mathrm{C}$ NMR spectra of synthesized molecular addition compounds $\mathbf{B}_{\mathbf{1}}-\mathbf{B}_{\mathbf{3}}$ are illustrated in Figures 5-7 and observed characteristic carbon chemical shifts $(\delta, \mathrm{ppm})$ are tabulated in Table 2.

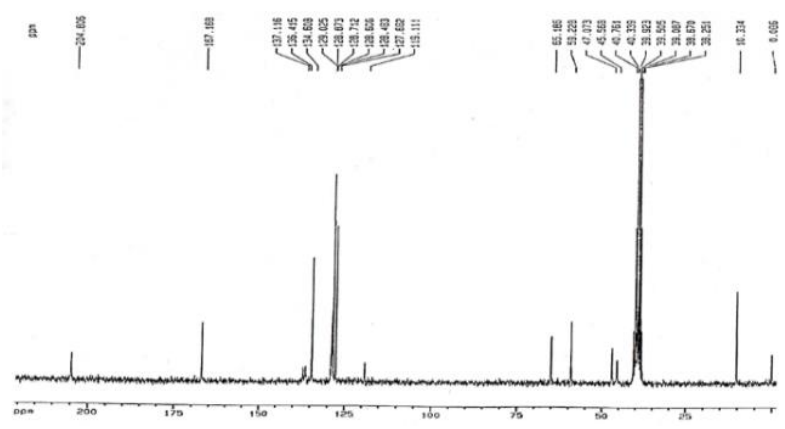

Figure 5. ${ }^{13} \mathrm{C}$ NMR spectrum of molecular addition compound $\mathbf{B}_{1}$

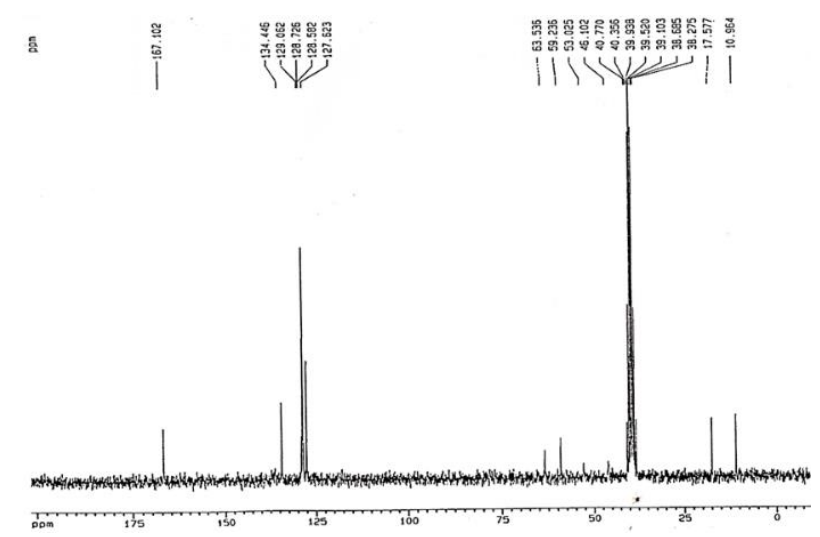

Figure 6. ${ }^{13} \mathrm{C}$ NMR spectrum of molecular addition compound $\mathbf{B}_{2}$ 


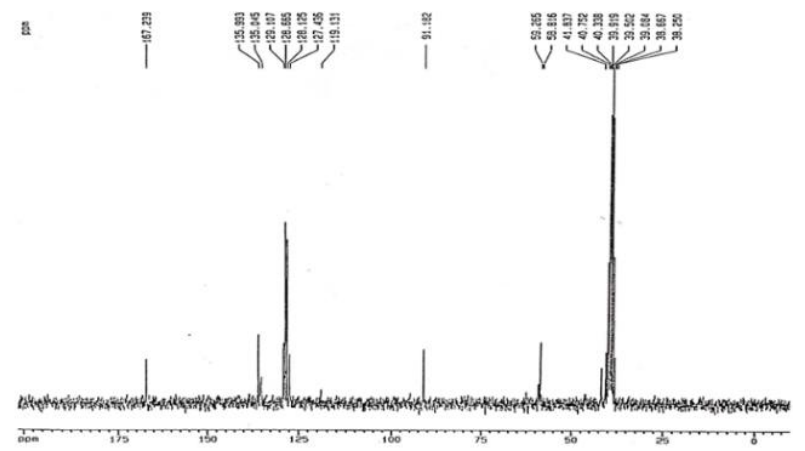

Figure 7. ${ }^{13} \mathrm{C}$ NMR spectrum of molecular addition compound $\mathbf{B}_{3}$

Table 2. The ${ }^{13} \mathrm{C}$ chemical shifts (ppm) of $\mathbf{B}_{\mathbf{1}}-\mathbf{B}_{\mathbf{3}}$ and the corresponding parents $\mathbf{A}_{\mathbf{1}}-\mathbf{A}_{\mathbf{3}}$

\begin{tabular}{|c|c|c|c|c|c|c|c|c|c|c|}
\hline \multirow[t]{2}{*}{ Cpds. } & \multirow[t]{2}{*}{$\mathrm{C}-2$} & \multirow[t]{2}{*}{$\mathrm{C}-3$} & \multirow[t]{2}{*}{ C-4 } & \multirow[t]{2}{*}{ C-5 } & \multirow[t]{2}{*}{ C-6 } & \multirow{2}{*}{$\begin{array}{l}\text { Alkyl } \\
\text { carbons }\end{array}$} & \multirow{2}{*}{$\begin{array}{l}\text { Aromatic } \\
\text { carbons }\end{array}$} & \multicolumn{2}{|c|}{ Ipso carbons } & \multirow{2}{*}{$\begin{array}{l}\text { Other } \\
\text { carbons }\end{array}$} \\
\hline & & & & & & & & $\mathrm{C}-1$ ' & C-1" & \\
\hline $\mathrm{B}_{1}$ & 65.2 & 47.1 & 204.8 & 45.6 & 59.2 & 10.3 & $127.7-134.6$ & 136.4 & 137.1 & $\begin{array}{l}167.2,134.6 \\
19.1\end{array}$ \\
\hline $\mathrm{B}_{2}$ & 63.5 & 53.0 & - & 46.1 & $\begin{array}{l}59.2 \\
45\end{array}$ & $\begin{array}{l}11.0\left(\mathrm{CH}_{3}\right) \\
17.6\left(\mathrm{CH}_{2}\right)\end{array}$ & $125.4-129.0$ & 137.1 & 138.4 & $167.2,134.5$ \\
\hline $\mathrm{B}_{3}$ & $\begin{array}{c}58.8 \\
(59.3)\end{array}$ & 53.0 & - & 41.8 & $\begin{array}{l}58.8 \\
(59.3)\end{array}$ & - & $127.4-129.1$ & 135.0 & 136.0 & $\begin{array}{l}\text { 167.2, 136.0, } \\
119.0,91.2\end{array}$ \\
\hline $\mathrm{A}_{1}$ & 68.4 & 51.6 & 209.5 & 50.3 & 61.5 & 10.1 & $127.9-128.6$ & 141.8 & 142.7 & - \\
\hline $\mathrm{A}_{2}$ & 66.7 & 58.4 & 209.1 & 51.6 & 61.8 & $\begin{array}{l}17.8\left(\mathrm{CH}_{2}\right) \\
12.2\left(\mathrm{CH}_{2}\right)\end{array}$ & $121.7-126.8$ & 141.7 & 143.0 & - \\
\hline $\mathrm{A}_{3}$ & 61.0 & 50.2 & 207.8 & 50.2 & 61.0 & - & $126.4-128.8$ & 142.6 & 142.6 & - \\
\hline
\end{tabular}

The chemical shifts (ppm) of aryl carbons are well illustrated by their typical absorption at about $120 \mathrm{ppm}$. The ipso carbons should absorb at downfield related to additional aromatic carbons. Assigned signals around $210 \mathrm{ppm}$ are due to $\mathrm{C}-4$ carbonyl group. The signals around $165 \mathrm{ppm}$ are due to carbonyl of maleic part of the compound. The upfield signals around $20 \mathrm{ppm}$ are dispensed to be alkyl carbons at C-3. The heterocyclic ring carbon absorptions were predicted based on known substituent possessions of alkyl group and on the basis of comparison of the signals with most of the parent piperidin-4-ones [14].

In the 3-methy 1 compound $\mathbf{B}_{1}$ the heterocyclic ring carbons resonate at $65.19,59.23,47.07$ and $45.58 \mathrm{ppm}$. Deshielded benzylic carbon signals were obtained at 65.19 and $59.23 \mathrm{ppm}$. Among these signals the one at downfield, i.e. $65.19 \mathrm{ppm}$, was dispensed to C-2. The $\mathrm{C}-3$ carbon chemical shifts experienced by the known $\beta$ effect of methyl group. Apparently, the remaining signals at $59.23 \mathrm{ppm}$ was outstanding to C-6. Among the remaining signals at 47.07 and $45.58 \mathrm{ppm}$ the one at downfield, i.e. $47.07 \mathrm{ppm}$, was dispensed for C-3. These allocations are created by the notorious $\alpha$-effect of methyl group at C-3. The signal at $45.58 \mathrm{ppm}$ is due to $\mathrm{C}-5$. The high intense peak $\mathrm{CH}$ carbons of the maleic part of the compound was obtained at $135.99 \mathrm{ppm}$.

In a similar manner, the assignments were made for 3-ethyl compound $\mathbf{B}_{2}$. For simple compound $\mathbf{B}_{\mathbf{3}}$ the ${ }^{13} \mathrm{C}$ NMR spectrum reveals two sets of signals for the heterocyclic ring carbons. The high intense signals at 58.82 and $41.83 \mathrm{ppm}$ are taken as the signals for the simple compound $\mathbf{B}_{3}$. The remaining signals are due to the signals for the other isomer present in the solution. Table 2 reports the ${ }^{13} \mathrm{C}$ data of addition compounds $\mathbf{B}_{1}$, $\mathbf{B}_{2}, \mathbf{B}_{3}$ and the corresponding piperidin-4-ones $\mathbf{A}_{1}, \mathbf{A}_{2}$, A3.

These are evident that the ${ }^{13} \mathrm{C}$ NMR data of all heterocyclic ring carbons, ipso carbons and alkyl carbons are shielded due to the formation of molecular addition compounds with maleic anhydride and are presented in Table 2, an exception being methyl carbon at C-3 where a small deshielding magnitude was detected. The magnitudes of observed shielding are calculated $\left(\delta_{\text {addition }}\right.$ compound $\left.-\delta_{\text {ketones }}\right)$ and these are tabulated in Table 3.

Table 3. The ${ }^{13} \mathrm{C}$ NMR chemical shifts ( $\mathrm{ppm}$ ) difference between of $\mathrm{B}_{1}-\mathrm{B}_{3}$ and their corresponding parents $\mathbf{A}_{1}-\mathbf{A}_{3}$

\begin{tabular}{|c|c|c|c|c|c|}
\hline Compounds & $\mathrm{C}-2$ & C-3 & C-4 & C-5 & C-6 \\
\hline $\mathbf{B}_{1}-\mathbf{A}_{1}$ & -3.2 & -4.5 & -4.7 & -5.3 & -2.3 \\
\hline $\mathbf{B}_{2}-\mathbf{A}_{2}$ & -3.2 & -5.4 & - & -5.5 & -2.6 \\
\hline $\mathbf{B}_{3}-\mathbf{A}_{3}$ & -3.2 & -4.3 & - & -4.3 & -3.2 \\
\hline
\end{tabular}


The benzylic protons skillfully deshielded by the steric interaction of the $\mathrm{CO}$ oxygen of the maleic anhydride portion of the addition compound. This interaction is probably lower when compared to syn 1,3diaxial interaction. In compound $\mathbf{B}_{1}$ the shielding magnitude detected on C-3 was much lesser than that of practical on $\mathrm{C}-5$. It is a well-known fact that flattening occurs near $C(2)-C(3)$ bond relative to $C(5)-C(6)$ bond in 3-alkyl-2,6-diphenylpiperidine derivatives, in order to decrease the phenyl-alkyl gauche interaction. The heterocyclic ring in $\mathbf{B}_{1}$ was highly flattened near $\mathrm{C}(2)$ $\mathrm{C}$ (3) when compared to 3-methylpiperidone $\mathbf{A}_{\mathbf{1}}$. In the flattened conformation more steric interaction is experienced on axial proton at $\mathrm{C}-5$ rather than the axial proton at $\mathrm{C}-3$. This is probably the reason for the higher magnitude of shielding observed on $\mathrm{C}(5)$ related to $\mathrm{C}(3)$ of $\mathbf{B}_{1}$.

The magnitudes of shielding observed on $\mathrm{C}(2)$ carbons are found to be the same in all the molecular addition compounds. However, the shielding magnitude detected on $\mathrm{C}(6)$ carbons are slightly lower than those on $\mathrm{C}(2)$ carbons. This is due to the deshielding of benzylic hydrogen and carbons by steric interaction. Pandiarajan and Manimekalai [20] have conveyed that the electronic effects of substituent are significantly altered owing to gauche interactions. In some cases, the magnitude and symbol of the $\alpha$-effect are altered by the presence of the neighboring substituent. In the molecular addition compound $\mathbf{B}_{2}$ probably the substituent effect of benzene moiety at C-2 is modified due to severe gauche interfacial interaction between phenyl and methyl group and between phenyl and maleic anhydride group. On the other hand, phenyl group at C-6 involves only the gauche interfacial interaction with the nearby maleic anhydride group. Gauche interaction is severe near $\mathrm{C}(2)-\mathrm{C}(3)$ side compared at $\mathrm{C}(5)-\mathrm{C}(6)$ side. Therefore, the $\alpha$-effect of benzene moiety was greatly decreased at $\mathrm{C}-2$ related to C-6. Hence large shielding magnitude is detected on C2 compared with C-6 in the molecular addition compounds.

\subsection{Mass spectrometry}

3.3.1. Mass spectral analysis of $B_{1}$ compound. Further the formation of compound $\mathbf{B}_{1}$ was confirmed by the mass spectrum illustrated in Figure 8.

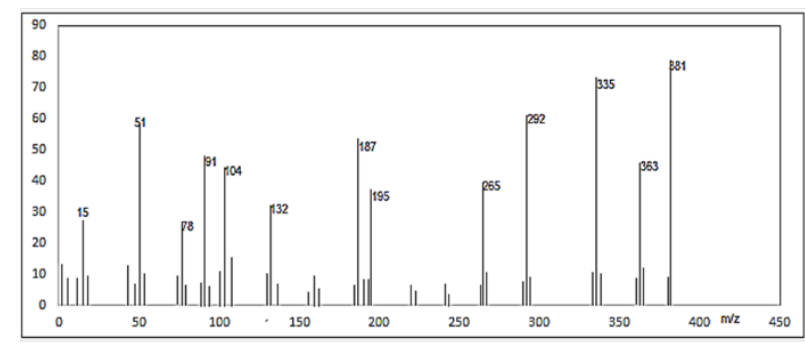

Figure 8. Mass spectrum of $\mathbf{B}_{\mathbf{1}}$

The important fragmentation patterns are shown in Fig. 9.

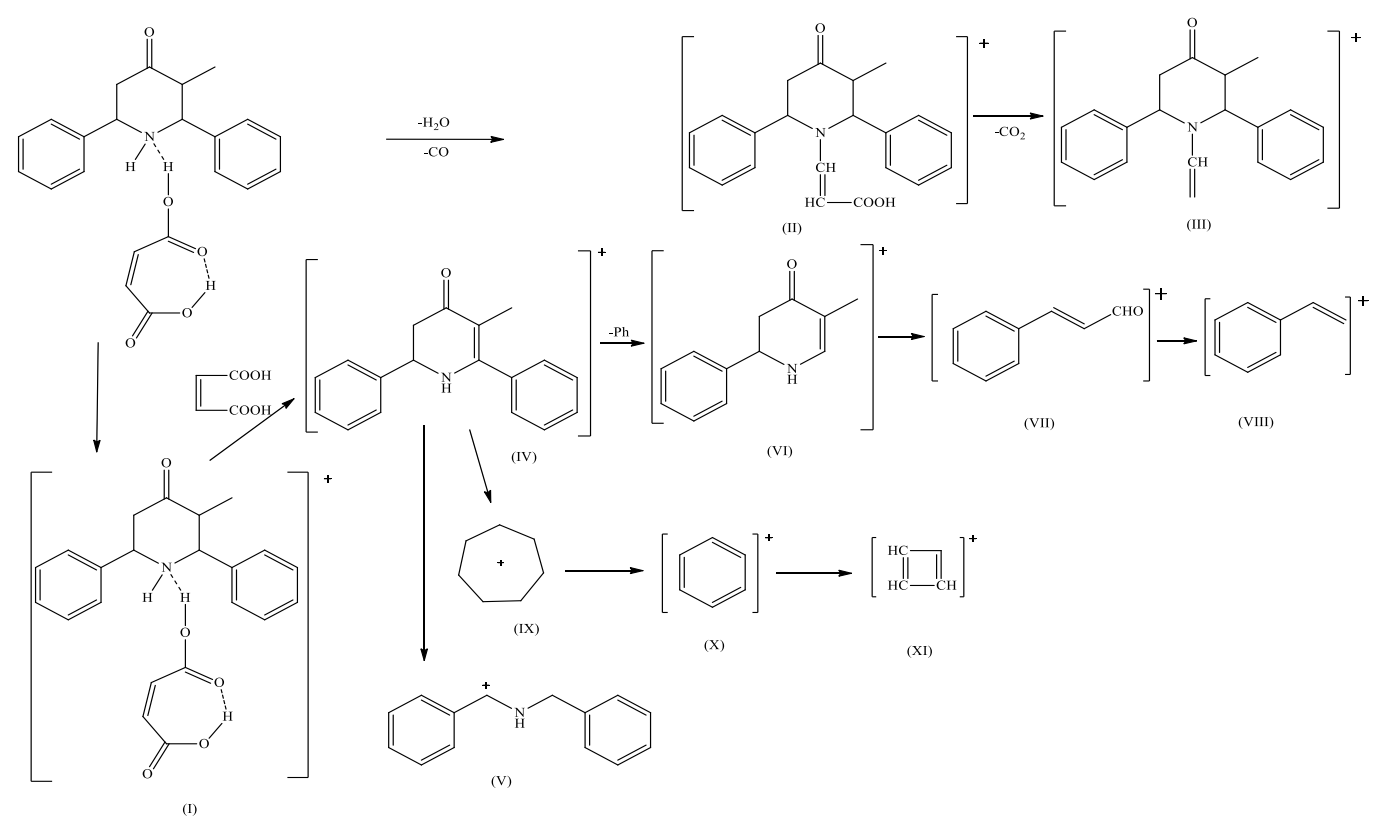

Figure 9. Fragmentation pattern of compound $\mathbf{B}_{\mathbf{1}}$

The presence of $\left(\mathrm{M}-\mathrm{H}_{2} \mathrm{O}\right)^{+} \mathrm{m} / \mathrm{z} 363$ (I) of compound B $_{1}$ suggests that the $\mathrm{H}_{2} \mathrm{O}$ molecule is lost very easily. The peak obtained at $\mathrm{m} / \mathrm{z} 335$ is owing to the species (II). The peak obtained at $\mathrm{m} / \mathrm{z} 292$ is owing to the cation (III) by the loss of $\mathrm{CO}_{2}$. The ion with mass 265 is most probably due to the 3-methylpiperidin-4-one (IV) cation. The peak obtained at $\mathrm{m} / \mathrm{z} 195$ is probably owing to the $\mathrm{Ph}-+\mathrm{CHNH}-\mathrm{CHPh}$ species (V). The peak obtained at $\mathrm{m} / \mathrm{z} 187$ (VI) is owing to loss of one benzene from 3-methylpiperidones. The peak obtained at $\mathrm{m} / \mathrm{z}$
132 is owing to the molecular ionic species (VII). The base peak appeared at $\mathrm{m} / \mathrm{z} 104$ (VIII) is owing to the styrene cation. Pandiarajan et al. [19] also reported the same fragmentation pattern for several 3-alkylpiperidine derivatives. The intense peak appeared at $\mathrm{m} / \mathrm{z} 91$ (IX) is owing to the tropylium cation. The peak obtained at $\mathrm{m} / \mathrm{z}$ 78 is probably owing to benzene molecular ion $(\mathbf{X})$. Obtained fragment $\mathrm{C}_{4} \mathrm{H}_{3}{ }^{+}$(XI) derived from benzene molecular ion is responsible for the peak at $\mathrm{m} / \mathrm{z} 51$. Thus, the compound $\mathbf{B}_{1}$ was formed by molecular 
addition method. In these cases, compounds resemble acid - base adduct only.

3.3.2. Mass spectral analysis of $B_{2}$ compound. The molecular adduct $\mathbf{B}_{\mathbf{2}}$ was further confirmed by mass spectrum shown in Figure 10.

The important fragmentation patterns are shown in Figure 11.

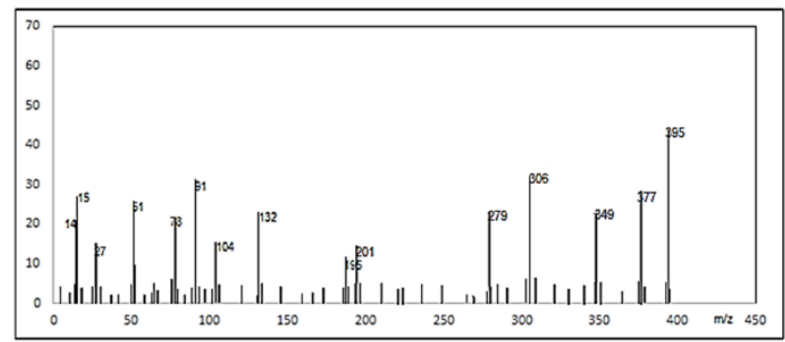

Figure 10. Mass spectrum of $\mathbf{B}_{2}$

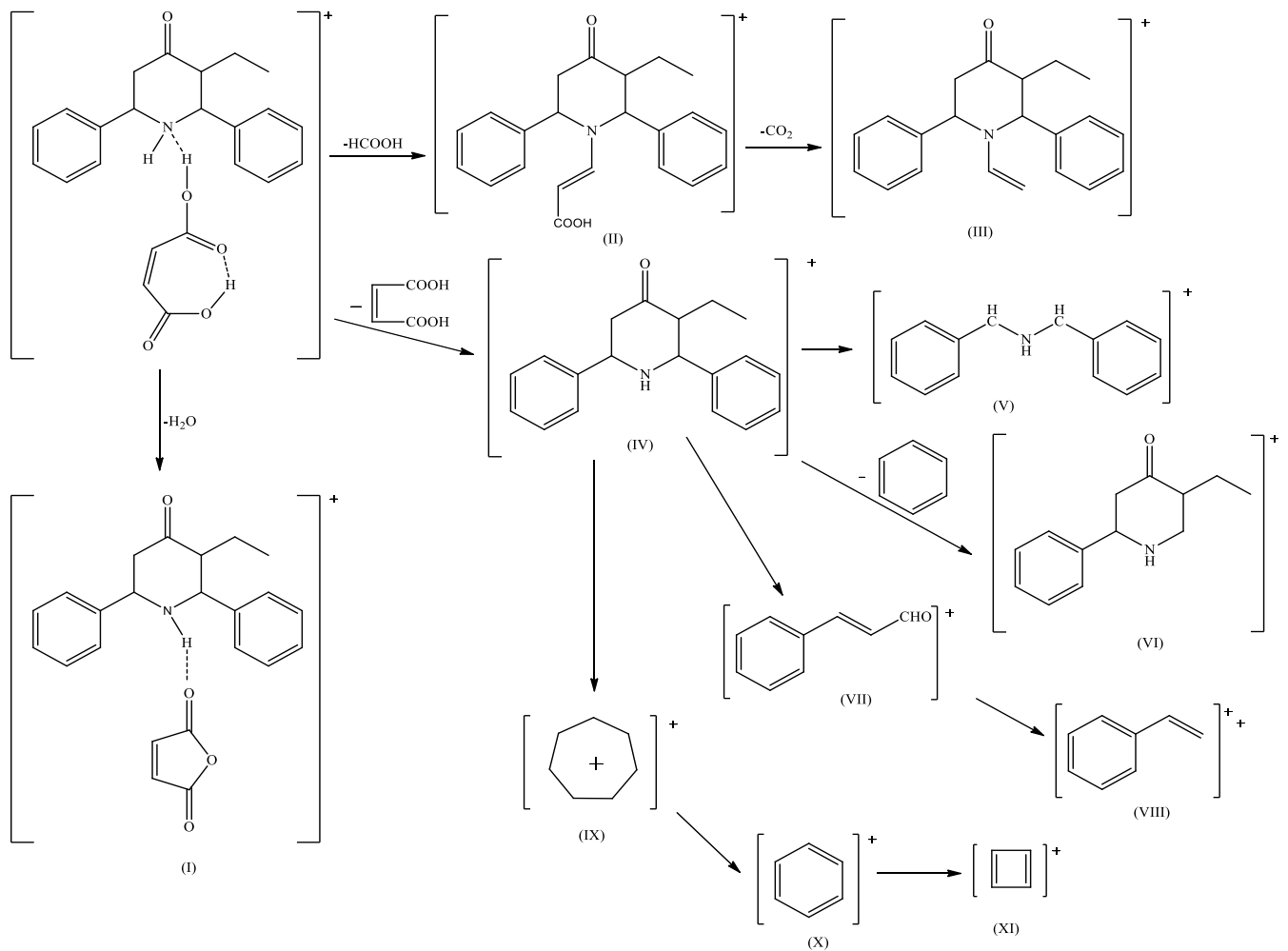

Figure 11. Fragmentation pattern of $\mathbf{B}_{2}$

The presence of $\left(\mathrm{M}-\mathrm{H}_{2} \mathrm{O}\right)^{+} \mathrm{m} / \mathrm{z} 377$ (I) of compound B2 suggests that the $\mathrm{H}_{2} \mathrm{O}$ molecule is lost very easily. The peak appeared at $\mathrm{m} / \mathrm{z} 349$ is owing to the species (II). The peak obtained at $\mathrm{m} / \mathrm{z} 306$ is owing to the cation (III) by the loss of $\mathrm{CO}_{2}$. The ion with mass 278 most probably corresponds to the 3 methylpiperidin-4-one cation (IV). The peak appeared at $\mathrm{m} / \mathrm{z} 195$ is probably owing to the $\mathrm{Ph}^{-}{ }^{+} \mathrm{CHNH}-\mathrm{CHPh}$ species $(\mathbf{V})$. The peak obtained at $\mathrm{m} / \mathrm{z} 200$ (VI) is owing to loss of one phenyl moiety from 3-ethylpiperidones. The peak appeared at $\mathrm{m} / \mathrm{z} 132$ was corresponding to the molecular ionic species (VII). The base peak obtained at m/z 104 (VIII) is owing to the styrene cation.

The peak obtained at $\mathrm{m} / \mathrm{z} 91$ (IX) corresponds to the tropylium cation. The peak appeared at $\mathrm{m} / \mathrm{z} 78$ is probably owing to benzene molecular ion $(\mathbf{X})$. Fragment $\mathrm{C}_{4} \mathrm{H}_{3}{ }^{+}$(XI) derived from benzene molecular ion is responsible for the peak obtained at $\mathrm{m} / \mathrm{z} 51$. Thus, the compound $\mathbf{B}_{2}$ was formed by molecular addition method. In this case, compound resemble acid - base adduct only.

3.3.3. Mass spectral analysis of $B_{3}$. The molecular addition compound $\mathbf{B}_{\mathbf{3}}$ was confirmed by mass spectrum shown in Figure 12.

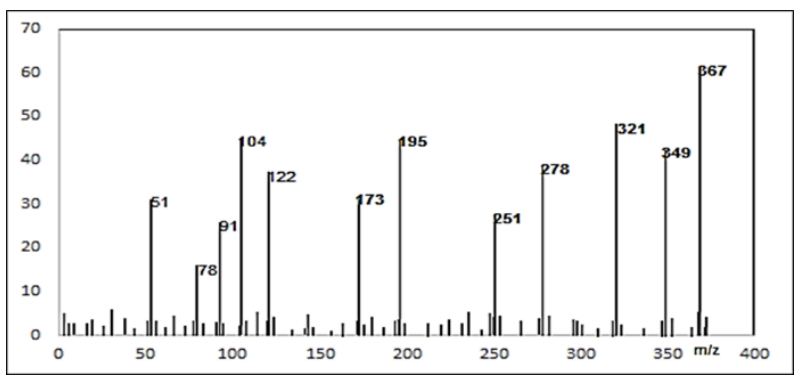

Figure 12. Mass spectrum of $\mathbf{B}_{3}$

The important fragmentation patterns are shown in Figure 13. 


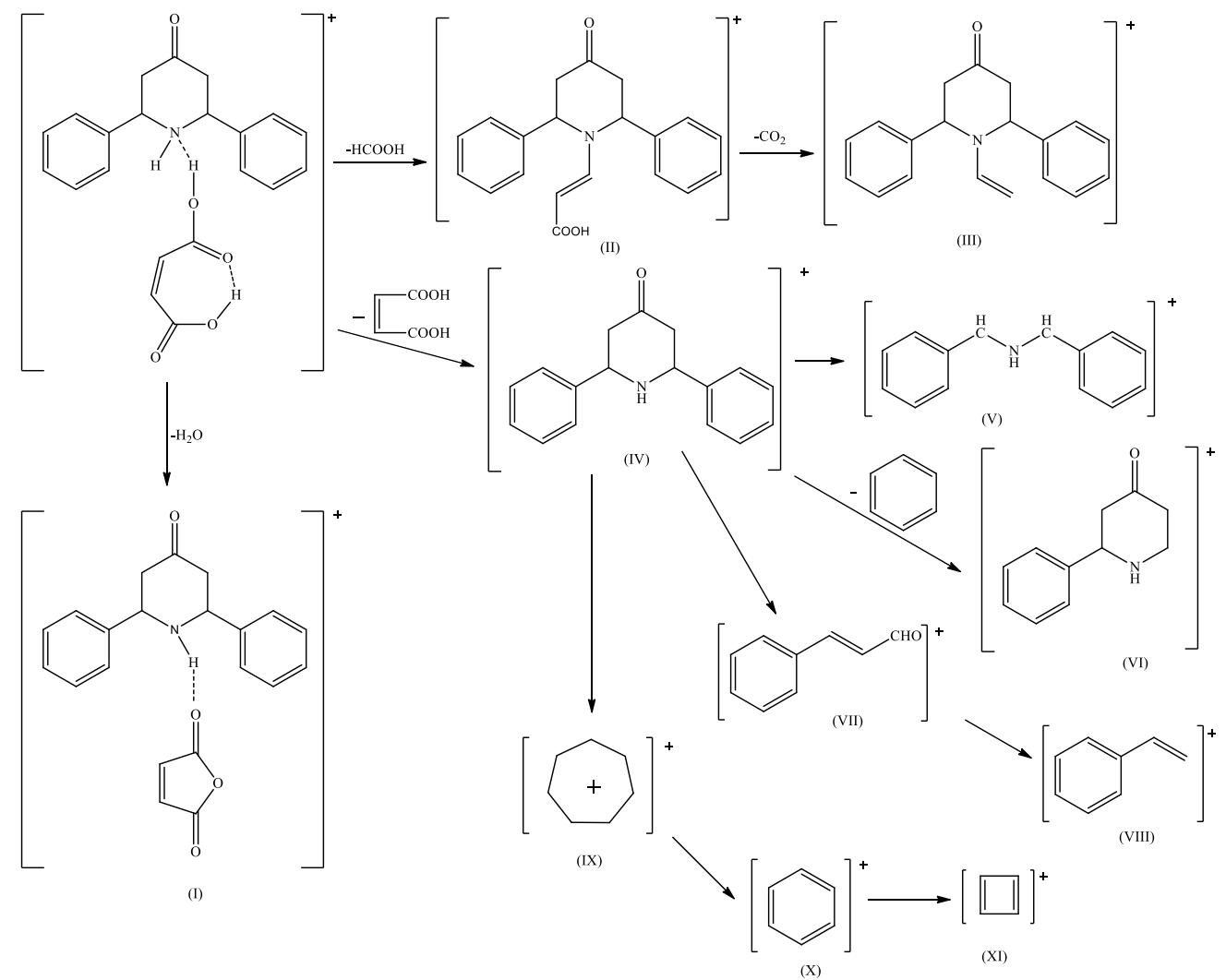

Figure 13. Fragmentation pattern of $\mathbf{B}_{3}$

The presence of $\left(\mathrm{m}-\mathrm{H}_{2} \mathrm{O}\right)^{+} \mathrm{m} / \mathrm{z} 349$ (I) of compound B 3 suggests that the $\mathrm{H}_{2} \mathrm{O}$ molecule is lost very easily. The peak appeared at $\mathrm{m} / \mathrm{z} 321$ corresponds to the species (II). The peak obtained at $\mathrm{m} / \mathrm{z} 278$ illustrated the fragment (III). The ion with mass 251 is most probably due to the 3-methylpiperidin-4-one (IV) cation. The peak appeared at $\mathrm{m} / \mathrm{z} 195$ is probably owing to the $\mathrm{Ph}^{-+}$ CHNH-CHPh species (V). The peak obtained at m/z 173 (VI) is corresponding to the loss of one benzene from piperidones. The peak obtained at $\mathrm{m} / \mathrm{z} 132$ was owing to the molecular ionic species (VII). The base peak appeared at $\mathrm{m} / \mathrm{z} 104$ (VIII) is owing to the styrene cation. The peak appeared at $\mathrm{m} / \mathrm{z} 91$ (IX) corresponds to the tropylium cation. The peak obtained at $(\mathrm{m} / \mathrm{z}) 78$ is probably owing to benzene molecular ion $(\mathbf{X})$. Fragment $\mathrm{C}_{4} \mathrm{H}_{3}{ }^{+}$(XI) derived from benzene molecular ion is responsible for the peak at $\mathrm{m} / \mathrm{z} 51$. Thus, the formed compound was only molecular addition compounds. In these cases, compounds resemble acid - base adduct only.

\section{Conclusions}

Molecular addition compounds $\mathbf{B}_{\mathbf{1}}, \mathbf{B}_{\mathbf{2}}$ and $\mathbf{B}_{\mathbf{3}}$ were synthesized by mixing maleic anhydride and piperidin4-ones $\mathbf{A}_{1}, \mathbf{A}_{\mathbf{2}}$ and $\mathbf{A}_{\mathbf{3}}$ in ether medium. The structures of compounds $\mathbf{B}_{1}, \mathbf{B}_{\mathbf{2}}$ and $\mathbf{B}_{\mathbf{3}}$ were explained. The NMR spectra of all synthesized compounds were recorded. The ${ }^{1} \mathrm{H}$ and ${ }^{13} \mathrm{C}$ NMR chemical shifts of adducts were analyzed by comparing with their initial piperidin-4ones. The chemical shifts assigned from ${ }^{1} \mathrm{H}$ and ${ }^{13} \mathrm{C}$ NMR spectra were strongly supported the fact that the product is not a N-substituted one, similar to $\mathrm{N}$ propanoyl or $\mathrm{N}$-acetylpiperidin-4-one. The ${ }^{1} \mathrm{H}$ NMR data revealed that there are considerable deshielding magnitude for all the heterocyclic protons. The ${ }^{13} \mathrm{C}$ chemical shifts of piperidone ring revealed that it is a considerable shielding magnitude for all compounds. The differences of ${ }^{13} \mathrm{C}$ chemical shifts between the compounds $\mathbf{B}_{\mathbf{1}}, \mathbf{B}_{\mathbf{2}}$, and $\mathbf{B}_{\mathbf{3}}$ and the corresponding parent piperidin-4- ones, $\mathbf{A}_{1}, \mathbf{A}_{\mathbf{2}}$ and $\mathbf{A}_{\mathbf{3}}$ are tabulated and discussed. Similarly, ${ }^{1} \mathrm{H}$ NMR chemical shift differences are also discussed in relation with the steric and polar effects. Mass spectrum of all adducts were recorded for $\mathbf{B}_{\mathbf{1}}-\mathbf{B}_{\mathbf{3}}$ and the fragmentation patterns were accounted.

\section{Acknowledgment}

Authors thank SAIF, IIT-Madras, Chennai-600 036 for recording NMR spectra of all compounds.

\section{Conflict of interest}

Authors declare no conflict of interest.

\section{References}

[1]. J.B. Lambert, D.A. Netzel, H.N. Sun, K.K. Lilianstrom, J. Am. Chem. Soc. 98 (1976) 3778.

[2]. K. Ramalingam, K.D. Berlin, N. Sathyamurthy, R. Sivakumar, J. Org. Chem. 44 (1979) 471.

[3]. T. Ravidran, R. Jeyaraman, R.W. Murray, M. Singh, J. Org. Chem. 56 (1991) 4833.

[4]. J.R. Dyer, Applications of Absorption Spectroscopy of Organic Compounds, Prentice Hall of India Private Limited, New Delhi, 1969.

[5]. A.W. Burgstabler, P.L. Chien, M.O. Abel Rahman, J. Am. Chem. Soc. 86 (1964) 5281.

[6]. W. Nagata, T. Terasawa, K. Tori, J. Am. Chem. Soc. 86 (1964) 3746. 
[7]. T. Schaefer, W.F. Reynolds, Y. Yonemato, Can. J. Chem. 4 (1963) 2969.

[8]. C. Hoogzard, W. Hubel, Tetrahedron Lett. 2 (1961) 637.

[9]. G. Slomp, B.K. Mc-Garvey, J. Am. Chem. Soc. 81 (1959) 2200.

[10]. A. M. Shestopalov, A. A. Shestopalov, L. A. Rodinovskaya, A. V. Gromova, Phosphorous, Sulfur, and Silicon and the Related Elements 184 (2009) 110.

[11]. M. Ul-Hasan, M. Arab, Magn, Reson. Chem. 25 (1987) 987.

[12]. G. Aridoss, S. Balasubramanian, P. Parthiban, S. Kabilan, Eur. J. Med. Chem. 42 (2007) 851.

[13]. K.K. Goel, A. Gajbhiye, Anu, N.M. Goel, Biomed. Pharmacol. J. 1 (2008) 201.

[14]. P. Krogsgaard-Larsen, E. Falch, A. Schousboe, D. R. Curtist, D. Lodget, J. Neurochem. 34 (1980) 756.
[15]. K.E. Panter, D.R. Gardner, R.J. Molyneux, J. Nat. Toxins 7 (1998) 131.

[16]. C. R. Noller, V. Baliah, J. Am. Chem. Soc. 70 (1948) 3853.

[17]. K. Lakshmanan, G. Thirunarayanan, World Scientific News 116 (2019) 102.

[18]. J. John Francis Xavier, K. Krishnasamy, C. Sankar, Med. Chem. Res. 21 (2012) 345.

[19]. K. Pandiarajan, K. Ganapathy, V. Gopalakrishnan, K. Ramalingam, Indian J. Heterocycl. Chem. 2 (1993) 159.

[20]. K. Pandiarajan, A. Manimekalai, Magn. Reason. Chem. 31 (1993) 80.

Received: 22.02 .2020

Received in revised form: 02.04.2020

Accepted: 08.04.2020 